\title{
In Plain Sight: Media Bias Through the Lens of Factual Reporting
}

\author{
Lisa Fan $^{1, *} \quad$ Marshall White $^{1, *} \quad$ Eva Sharma $^{1} \quad$ Ruisi Su $^{1}$ \\ Prafulla Kumar Choubey $^{2} \quad$ Ruihong Huang $^{2} \quad$ Lu Wang $^{1}$ \\ ${ }^{1}$ Khoury College of Computer Sciences, Northeastern University, Boston, MA 02115 \\ ${ }^{2}$ Department of Computer Science and Engineering, Texas A\&M University \\ \{fan.lis, white.mars, sharma.ev, su.ruis\}@husky.neu.edu \\ \{prafulla.choubey, huangrh\}etamu.edu, luwangeccs.neu.edu
}

\begin{abstract}
The increasing prevalence of political bias in news media calls for greater public awareness of it, as well as robust methods for its detection. While prior work in NLP has primarily focused on the lexical bias captured by linguistic attributes such as word choice and syntax, other types of bias stem from the actual content selected for inclusion in the text. In this work, we investigate the effects of informational bias: factual content that can nevertheless be deployed to sway reader opinion. We first produce a new dataset, BASIL, of 300 news articles annotated with 1,727 bias spans ${ }^{1}$ and find evidence that informational bias appears in news articles more frequently than lexical bias. We further study our annotations to observe how informational bias surfaces in news articles by different media outlets. Lastly, a baseline model for informational bias prediction is presented by finetuning BERT on our labeled data, indicating the challenges of the task and future directions.
\end{abstract}

\section{Introduction}

News media exercises the vast power of swaying public opinion through the way it selects and crafts information (De Vreese, 2004; DellaVigna and Gentzkow, 2010; McCombs and Reynolds, 2009; Perse, 2001; Reynolds and McCombs, 2002). Multiple studies have identified the correlation between the increasing polarization of media and the general population's political stance (Gentzkow and Shapiro, 2010, 2011; Prior, 2013), underscoring the imperative to understand the nature of news bias and how to accurately detect it.

In the natural language processing community, the study of bias has centered around what we term

\footnotetext{
${ }^{*}$ Equal contribution. Lisa Fan focused on annotation schema design and writing, Marshall White focused on data collection and statistical analysis.

${ }^{1}$ Dataset can be found at www. ccs. neu.edu/home/ luwang/data.html.
}

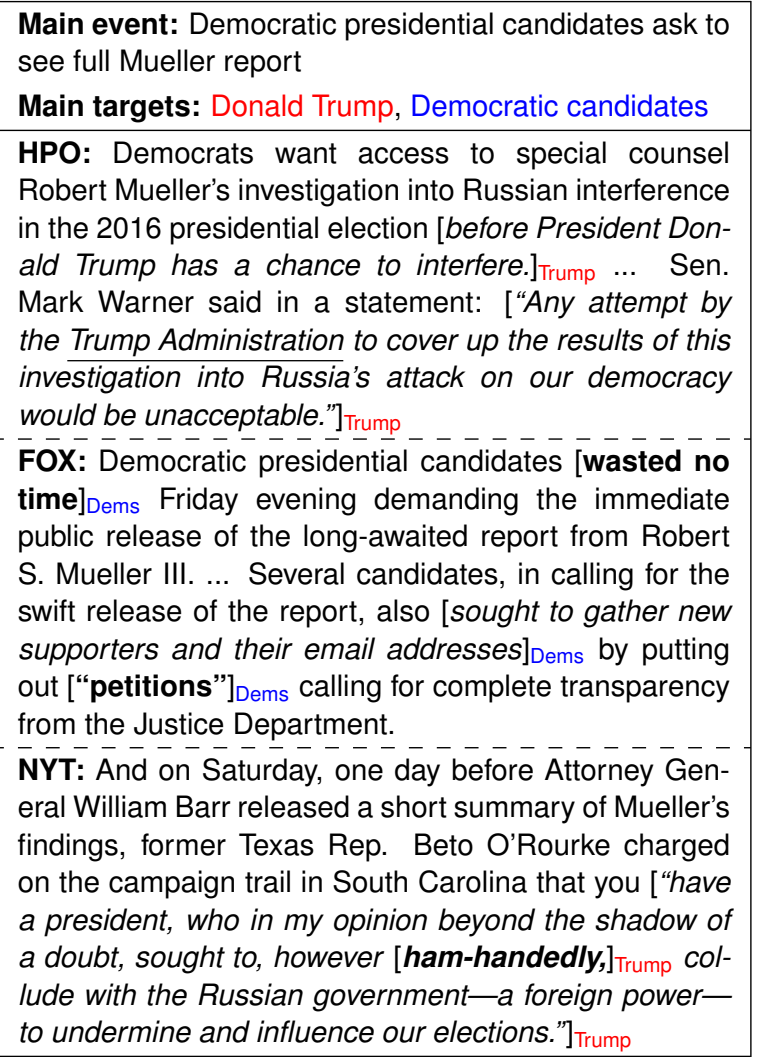

Figure 1: Examples of negative bias from Huffington Post (HPO), Fox News (FOX), and New York Times (NYT) discussing the same event. Informational bias and lexical bias are highlighted. The target of the bias is noted at the end of each span. Intermediary targets of indirect bias spans are underlined.

lexical bias: bias stemming from content realization, or how things are said (Greene and Resnik, 2009; Hube and Fetahu, 2019; Iyyer et al., 2014; Recasens et al., 2013; Yano et al., 2010). Such forms of bias typically do not depend on context outside of the sentence and can be alleviated while maintaining its semantics: polarized words can be removed or replaced, and clauses written in active voice can be rewritten in passive voice.

However, political science researchers find that news bias can also be characterized by decisions 
made regarding content selection and organization within articles (Gentzkow et al., 2015; Prat and Strömberg, 2013). As shown in Figure 1, though all three articles report on the same event, Huffington Post (HPO) and Fox News (FOX) each frame entities of opposing stances negatively: HPO states an assumed future action of Donald Trump as a fact, and FOX implies Democrats are taking advantage of political turmoil. Such bias can only be revealed by gathering information from a variety of sources or by analyzing how an entity is covered throughout the article.

We define these types of bias as informational bias: sentences or clauses that convey information tangential, speculative, or as background to the main event in order to sway readers' opinions towards entities in the news. Informational bias often depends on the broader context of an article, such as in the second FOX annotation in Figure 1: gathering new supporters would be benign in an article describing political campaign efforts. The subtlety of informational bias can more easily affect an unsuspecting reader, which presents the necessity of developing novel detection methods.

In order to study the differences between these two types of bias, we first collect and label a dataset, BASIL (Bias Annotation $\underline{\text { Spans on the }}$ Informational Level), of 300 news articles with lexical and informational bias spans. To examine how media sources encode bias differently, the dataset uses 100 triplets of articles, each reporting the same event from three outlets of different ideology. Based on our annotations, we find that all three sources use more informational bias than lexical bias, and informational bias is embedded uniformly across the entire article, while lexical bias is frequently observed at the beginning.

We further explore the challenges in bias detection and benchmark BASIL using rule-based classifiers and the BERT model (Devlin et al., 2019) fine-tuned on our data. Results show that identifying informational bias poses additional difficulty and suggest future directions of encoding contextual knowledge from the full articles as well as reporting by other media.

\section{Related Work}

Prior work on automatic bias detection based on natural language processing methods primarily deals with finding sentence-level bias and considers linguistic attributes like word polarity (Re- casens et al., 2013), partisan phrases (Yano et al., 2010), and verb transitivity (Greene and Resnik, 2009). However, such studies fail to take into consideration biases that depend on a larger context, which is what we try to address in this work.

Our work is also in line with framing analysis in social science theory, or the concept of selecting and signifying specific aspects of an event to promote a particular interpretation (Entman, 1993). In fact, informational bias can be considered a specific form of framing where the author intends to influence the reader's opinion of an entity. The relationship between framing and news is investigated by Card et al. (2015), in which news articles are annotated with framing dimensions like "legality" and "public opinion." BASIL contains richer information that allows us to study the purpose of "frames," i.e., how biased content is invoked to support or oppose the issue at hand.

Research in political science has also studied bias induced by the inclusion or omission of certain facts (Entman, 2007; Gentzkow and Shapiro, 2006, 2010; Prat and Strömberg, 2013). However, their definition of bias is typically grounded in how a reader perceives the ideological leaning of the article and news outlet, whereas our informational bias centers around the media's sentiment towards individual entities. Furthermore, while previous work mostly uses all articles published by a news outlet to estimate their ideology (Budak et al., 2016), we focus on stories of the same events reported by different outlets.

\section{BASIL Dataset Annotation}

Using a combination of algorithmic alignment and manual inspection, we select 100 sets of articles, each set discussing the same event from three different news outlets. 10 sets are selected for each year from 2010 to 2019 . We use, in order from most conservative to most liberal, Fox News (FOX), New York Times (NYT), and Huffington Post (HPO). Main events and main entities are manually identified for each article prior to annotation. The political leanings of the main entities (liberal, conservative, or neutral) are also manually annotated. See the Supplementary for details.

Annotation Process. To compare how the three media sources discuss a story, annotators treat each article triplet as a single unit without knowing media information. Annotations are conducted on both document-level and sentence-level. On 


\begin{tabular}{|c|c|c|c|c|c|}
\hline & NYT & FOX & HPO & All \\
\hline \multicolumn{2}{|c|}{ \# Articles } & 100 & 100 & 100 & 300 \\
\hline \multicolumn{2}{|c|}{ \# Sentences } & 3,049 & 2,639 & 2,296 & 7,984 \\
\hline \multicolumn{2}{|c|}{ \# Words } & 91,818 & 70,024 & 62,321 & 224,163 \\
\hline \multicolumn{2}{|c|}{ \# Annotations } & 636 & 573 & 518 & 1,727 \\
\hline \multicolumn{2}{|c|}{ Sentences / Article } & $30.5 \pm 13.8$ & $26.4 \pm 10.2$ & $23.0 \pm 11.0$ & $26.6 \pm 12.2$ \\
\hline \multicolumn{2}{|c|}{ Words / Sentence } & $30.1 \pm 14.0$ & $26.5 \pm 12.4$ & $27.1 \pm 12.5$ & $28.1 \pm 13.2$ \\
\hline \multicolumn{2}{|c|}{ Annotations / Article } & $6.4 \pm 4.1$ & $5.7 \pm 3.8$ & $5.2 \pm 3.5$ & $5.8 \pm 3.8$ \\
\hline \multirow{2}{*}{ Bias Type } & Informational & $468(73.6 \%)$ & $421(73.5 \%)$ & $360(69.5 \%)$ & $1,249(72.3 \%)$ \\
\hline & Lexical & $168(26.4 \%)$ & $152(26.5 \%)$ & $158(30.5 \%)$ & $478(27.7 \%)$ \\
\hline \multirow{2}{*}{ Aim } & Direct & $574(90.2 \%)$ & $485(84.6 \%)$ & $462(89.2 \%)$ & $1,521(88.1 \%)$ \\
\hline & Indirect & $62(9.8 \%)$ & $88(15.4 \%)$ & $56(10.8 \%)$ & $206(11.9 \%)$ \\
\hline \multirow{2}{*}{ Polarity } & Positive & $112(17.6 \%)$ & $89(15.5 \%)$ & $110(21.2 \%)$ & $311(18.0 \%)$ \\
\hline & Negative & $524(82.4 \%)$ & $484(84.5 \%)$ & $408(78.8 \%)$ & $1,416(82.0 \%)$ \\
\hline \multicolumn{2}{|c|}{ Annotations in quotes } & $205(32.2 \%)$ & $299(52.2 \%)$ & $217(41.9 \%)$ & $721(41.8 \%)$ \\
\hline
\end{tabular}

Table 1: Descriptive statistics of the BASIL dataset. Mean and standard deviation shown where applicable. Annotation dimensions show raw counts and their percentage within the dimension in parentheses.

the document-level, annotators estimate the overall polarities of how the main event and main entities are covered, and rank the triplet's articles on the ideological spectrum with respect to one another. Before reading the articles, annotators specify their sentiment towards each main entity on a 5 point Likert scale. ${ }^{2}$

On the sentence-level, annotators identify spans of lexical and informational bias by analyzing whether the text tends to affect a reader's feeling towards one of the main entities. In addition to the main dimension of bias type (lexical or informational), each span is labeled with the target of the bias (a main entity), the bias polarity (positive or negative towards the target), the bias aim towards the main target (direct or indirect), and whether the bias is part of a quote. Bias aim investigates the case where the main entity is indirectly targeted through an intermediary figure (see the HPO example in Figure 1, where the sentiment towards the intermediary entity "Trump Administration" is transferred to the main target, "Donald Trump"). Statistics are presented in Table 1.

Inter-annotator Agreement (IAA). Two annotators individually annotate each article triplet before discussing their annotations together to resolve conflicts and agree on "gold-standard" labels. We measure span-level agreement according to Toprak et al. (2010), where we calculate the F1 score of span overlaps between two sets of annotations (details are in the Supplementary). Although the F1 scores of IAA are unsurprisingly low for this highly variable task, the score dramatically in-

\footnotetext{
${ }^{2}$ The likely effect of annotators' prior beliefs on their perception of bias will be investigated in future work.
}

creases when agreement is calculated between individual annotations and the gold standard-from 0.34 to 0.70 for informational bias spans and from 0.14 to 0.56 for the sparser lexical spans, demonstrating the effectiveness of resolution discussions.

During the discussions, we noticed several trends that improved the quality of the gold standard annotations. First, the difficulty of being continually vigilant of one's own implicit bias would sometimes cause annotators to mark policies they disagreed with as negative bias (e.g., a liberal annotator might consider the detail that a politician supports an anti-abortion law as negative bias). Discussions allowed annotators to re-examine the articles from a more neutral perspective. Annotators also disagreed on whether a detail was relevant background or biasing peripheral information. During discussions, they performed comparisons to other articles of the triplet to make a final decision-if another article includes the same information, it is likely relevant to the main event. This strategy reiterates the importance of leveraging different media sources.

For overlapping spans, we find high agreement on the other annotation dimensions, with an average Cohen's $\kappa$ of 0.84 for polarity and 0.92 for target main entity.

\section{Media Bias Analysis}

\subsection{Contrasting the Bias Types}

Informational bias outnumbers lexical bias. As shown in Table 1, the large majority of annotations in BASIL are classified as informational bias. One explanation for its prevalence is that journalists typically make a conscious effort to avoid 

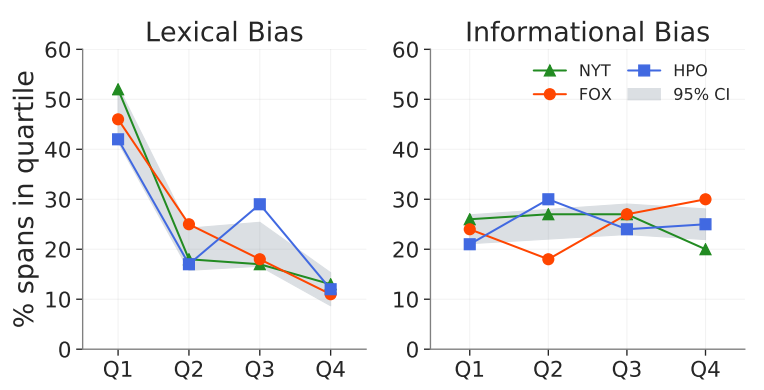

Figure 2: Distribution of lexical and informational bias spans found in each quartile of an article. The shaded area represents the $95 \%$ confidence interval for the three outlets combined.

biased language, but can still introduce informational bias, either intentionally or through negligence.

For both bias types though, negative bias spans are much more pervasive than positive spans, mirroring the well-established paradigm that news media in general focuses on negative events (Niven, 2001; Patterson, 1996).

Lexical bias appears early in an article. We further study differences in characteristics between lexical and informational annotation spans and find that the two bias types diverge in positional distributions. Figure 2 shows that a disproportionate amount of lexical bias is located in the first quartile of articles. A visual inspection indicates that this may be attributed in part to media sources' attempts to hook readers with inflammatory speech early on (e.g., FOX: "Paul Ryan stood his ground against a barrage of Biden grins, guffaws, snickers and interruptions.").

In contrast, informational bias is often embedded in context, and therefore can appear at any position in the article. This points to a future direction of bias detection using discourse analysis.

Quotations introduce informational bias. We also find that almost half of the informational bias comes from within quotes (48.7\%), highlighting a bias strategy where media sources select opinionated quotes as a subtle proxy for their own opinions (see the second HPO and first NYT annotations in Figure 1).

\subsection{Portrayal of Political Entities}

On the document-level, only 17 out of 100 article sets had reversed orderings (i.e. FOX marked as "more liberal" or HPO marked as "more conservative" within a triplet), confirming the ideological leanings identified in previous studies. Here,

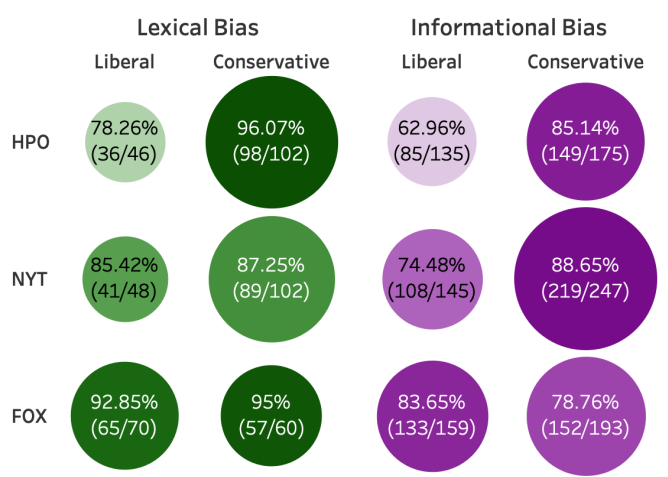

Figure 3: Percentage of bias spans with negative polarity toward targets of known ideology, grouped by media source, bias type, and target's ideology. For example, in all HPO articles, there are 46 lexical bias spans targeting liberals, $78.26 \%$ of which are negative. Larger circle means greater number of spans. Darker color corresponds to higher ratio of negative spans.

we utilize BASIL's span-level annotations to gain a more granular picture of how sources covering the same events control the perception of entities.

Concretely, we examine the polarity of bias spans with target entities of known ideology. As shown in Figure 3, for both bias types, the percentage and volume of negative coverage for liberal entities strongly correspond to the ideological leaning of the news outlet. Note that though NYT appears to have significantly more informational bias spans against conservatives than HPO, this is because NYT tends to have longer articles than the other two sources (see Table 1), and thus naturally results in more annotation spans by raw count. ${ }^{3}$

Moreover, the breakdown of lexical bias distinguishes FOX from the other two outlets: it comparatively has more negative bias spans towards liberals and fewer towards conservatives, even though all three outlets have more conservative entities than liberal ones across the 100 triplets (average of 99.0 conservatives, 72.7 liberals).

\section{Experiments on Bias Detection}

We study the bias prediction problem on BASIL as a binary classification task (i.e., whether or not a sentence contains bias) and as a BIO sequence tagging task (i.e., tagging the bias spans in one sentence at the token-level). We benchmark the performance with rule-based classifiers and the popular BERT model (Devlin et al., 2019) fine-tuned

\footnotetext{
${ }^{3}$ The proportion of annotations to article length are similar for all news outlets: one annotation for every 4.1 (for HPO), 4.5 (for FOX), or 4.6 (for NYT) sentences.
} 


\begin{tabular}{|lrrc|}
\hline Sentence-level & Precision & Recall & F1 \\
\hline Lexical Bias & & & \\
BERT fine-tuning & 29.13 & 38.57 & 31.49 \\
\hline Informational Bias & & & \\
TF-IDF & 25.81 & 26.23 & 26.02 \\
BERT fine-tuning & 43.87 & 42.91 & 43.27 \\
\hline
\end{tabular}

\begin{tabular}{|lrrr|}
\hline Token-level & Precision & Recall & \multicolumn{1}{c|}{ F1 } \\
\hline Lexical Bias & & & \\
Polarity lexicon & 8.00 & 0.17 & 0.33 \\
Subjectivity lexicon & 28.00 & 0.65 & 1.28 \\
BERT fine-tuning & 25.60 & 29.32 & 25.98 \\
\hline Informational Bias & & & \\
BERT fine-tuning & 25.56 & 14.78 & 18.71 \\
\hline Sentence-_o-Token pipeline ---- & & \\
Lexical bias & 12.00 & 13.64 & 12.77 \\
Informational bias & 9.52 & 5.08 & 6.63 \\
\hline
\end{tabular}

Table 2: Sentence classification (top) and sequence tagging (bottom) results on lexical and informational bias prediction. For the BERT fine-tuning models, the mean from 10 -fold cross validation is shown. The minimum standard deviation from cross validation for all BERT models is 3.36 , the maximum is 12.44 .

on informational and lexical bias spans separately.

Training Details. We utilize the pre-trained BERT-Base model and use the "Cased" version to account for named entities, which are important for bias detection. We run BERT on individual sentences ${ }^{4}$ and perform stratified 10-fold cross validation. The validation set is used to determine when to stop training and a held out test set is used for the final evaluation of each fold. For the sentence-level classifiers, both our informational and lexical models use 6,819 sentences for training, 758 for validation, and 400 for testing.

Due to the sparsity of our data, we train and test our token-level models only on sentences containing bias spans of the relevant bias type. Our informational and lexical bias sequence taggers use a train/val/test split of 1,043/116/62 sentences and 383/42/23 sentences respectively. Results are shown in Table 2.

Sentence-level Classifier. The fine-tuned BERT is better at predicting informational bias than lexical bias, likely because informational bias is better captured by sentence-level context. As a baseline, we select the 4 sentences ${ }^{5}$ in each article with the lowest average TF-IDF token scores as containing

\footnotetext{
${ }^{4}$ BERT's maximum input length is 512 tokens, which is shorter than most articles in BASIL. We thus treat sentences as passages, rather than using text of fixed length.

${ }^{5}$ BASIL averages 4.1 informational bias spans per article.
}

informational bias. The intuition is that sentences with different content than the rest of the article are more likely to contain extraneous information that the author chose to include to frame the story in a certain way. We find that this simple baseline performs relatively well considering the difficulty of the task, indicating the importance of explicitly modeling context. Future work may consider leveraging context in the entire article or articles on the same story by other media.

Token-level Classifier. From Table 2, we see that the BERT lexical sequence tagger produces better recall and F1 than the informational tagger, highlighting the additional difficulty of accurately identifying spans of informational bias. We also use the polarity and subjectivity lexicons from the MPQA website (Wilson et al., 2005; Choi and Wiebe, 2014) as a simple baseline for lexical bias tagging and find that these word-level cues, though widely used in prior sentiment analysis studies, are insufficient to fully capture lexical bias.

In order to evaluate token-level prediction on the larger original test set, we conduct a pipeline experiment with the fine-tuned BERT models where sentences predicted as containing bias by the best sentence-level classifier from cross validation are tagged by the best token-level model. The results reaffirm our hypothesis that while both tasks are extremely difficult, informational bias is more challenging to detect.

\section{Conclusion}

We presented a novel study on the effects of informational bias in news reporting from three major media outlets of different political ideology. Analysis of our annotated dataset, BASIL, showed the prevalence of informational bias in news articles when compared to lexical bias, and demonstrated BASIL's utility as a fine-grained indicator of how media outlets cover political figures. An experiment on bias prediction illustrated the importance of context when detecting informational bias and revealed future research directions.

\section{Acknowledgements}

This research is supported in part by National Science Foundation through Grant IIS-1813341. We thank Philip Resnik, Nick Beauchamp, and Donghee Jo for their valuable suggestions on various aspects of this work. We are also grateful to the anonymous reviewers for their comments. 


\section{References}

Ceren Budak, Sharad Goel, and Justin M Rao. 2016. Fair and balanced? quantifying media bias through crowdsourced content analysis. Public Opinion Quarterly, 80(S1):250-271.

Dallas Card, Amber E Boydstun, Justin H Gross, Philip Resnik, and Noah A Smith. 2015. The media frames corpus: Annotations of frames across issues. In Proceedings of the 53rd Annual Meeting of the Association for Computational Linguistics and the 7th International Joint Conference on Natural Language Processing (Volume 2: Short Papers), volume 2, pages 438-444.

Yoonjung Choi and Janyce Wiebe. 2014. +/effectwordnet: Sense-level lexicon acquisition for opinion inference. In Proceedings of the 2014 Conference on Empirical Methods in Natural Language Processing (EMNLP), pages 1181-1191.

Claes De Vreese. 2004. The effects of strategic news on political cynicism, issue evaluations, and policy support: A two-wave experiment. Mass Communication \& Society, 7(2):191-214.

Stefano DellaVigna and Matthew Gentzkow. 2010. Persuasion: empirical evidence. Annu. Rev. Econ., 2(1):643-669.

Jacob Devlin, Ming-Wei Chang, Kenton Lee, and Kristina Toutanova. 2019. Bert: Pre-training of deep bidirectional transformers for language understanding. In Proceedings of the 2019 Conference of the North American Chapter of the Association for Computational Linguistics: Human Language Technologies, Volume 1 (Long and Short Papers), pages 4171-4186.

Robert M Entman. 1993. Framing: Toward clarification of a fractured paradigm. Journal of communication, 43(4):51-58.

Robert M Entman. 2007. Framing bias: Media in the distribution of power. Journal of communication, 57(1):163-173.

Matthew Gentzkow and Jesse M Shapiro. 2006. Media bias and reputation. Journal of political Economy, 114(2):280-316.

Matthew Gentzkow and Jesse M Shapiro. 2010. What drives media slant? evidence from us daily newspapers. Econometrica, 78(1):35-71.

Matthew Gentzkow and Jesse M Shapiro. 2011. Ideological segregation online and offline. The Quarterly Journal of Economics, 126(4):1799-1839.

Matthew Gentzkow, Jesse M Shapiro, and Daniel F Stone. 2015. Media bias in the marketplace: Theory. In Handbook of media economics, volume 1, pages 623-645. Elsevier.
Stephan Greene and Philip Resnik. 2009. More than words: Syntactic packaging and implicit sentiment. In Proceedings of human language technologies: The 2009 annual conference of the north american chapter of the association for computational linguistics, pages 503-511. Association for Computational Linguistics.

Christoph Hube and Besnik Fetahu. 2019. Neural based statement classification for biased language. In Proceedings of the Twelfth ACM International Conference on Web Search and Data Mining, pages 195-203. ACM.

Mohit Iyyer, Peter Enns, Jordan Boyd-Graber, and Philip Resnik. 2014. Political ideology detection using recursive neural networks. In Proceedings of the 52nd Annual Meeting of the Association for Computational Linguistics (Volume 1: Long Papers), volume 1, pages 1113-1122.

Maxwell McCombs and Amy Reynolds. 2009. How the news shapes our civic agenda. In Media effects, pages 17-32. Routledge.

David Niven. 2001. Bias in the news: Partisanship and negativity in media coverage of presidents george bush and bill clinton. Harvard International Journal of Press/Politics, 6(3):31-46.

Thomas E Patterson. 1996. Bad news, bad governance. The Annals of the American Academy of Political and Social Science, 546(1):97-108.

Elizabeth M Perse. 2001. Media effects and society. Routledge.

Andrea Prat and David Strömberg. 2013. The political economy of mass media. Advances in economics and econometrics, 2:135.

Markus Prior. 2013. Media and political polarization. Annual Review of Political Science, 16:101-127.

Marta Recasens, Cristian Danescu-Niculescu-Mizil, and Dan Jurafsky. 2013. Linguistic models for analyzing and detecting biased language. In Proceedings of the 51st Annual Meeting of the Association for Computational Linguistics (Volume 1: Long Papers), volume 1, pages 1650-1659.

Amy Reynolds and Maxwell McCombs. 2002. News influence on our pictures of the world. In Media effects, pages 11-28. Routledge.

Cigdem Toprak, Niklas Jakob, and Iryna Gurevych. 2010. Sentence and expression level annotation of opinions in user-generated discourse. In Proceedings of the 48th Annual Meeting of the Association for Computational Linguistics, pages 575-584. Association for Computational Linguistics.

Theresa Wilson, Janyce Wiebe, and Paul Hoffmann. 2005. Recognizing contextual polarity in phraselevel sentiment analysis. In Proceedings of Human Language Technology Conference and Conference 
on Empirical Methods in Natural Language Processing.

Tae Yano, Philip Resnik, and Noah A Smith. 2010. Shedding (a thousand points of) light on biased language. In Proceedings of the NAACL HLT 2010 Workshop on Creating Speech and Language Data with Amazon's Mechanical Turk, pages 152-158. Association for Computational Linguistics. 